\title{
Twist-Bend Coupling and the Torsional Response of Double-Stranded DNA
}

\author{
Stefanos K. Nomidis, ${ }^{1,2}$ Franziska Kriegel, ${ }^{3}$ Willem Vanderlinden,,${ }^{3,4}$ Jan Lipfert, ${ }^{3}$ and Enrico Carlon ${ }^{1}$ \\ ${ }^{1}$ KU Leuven, Institute for Theoretical Physics, Celestijnenlaan 200D, 3001 Leuven, Belgium \\ ${ }^{2}$ Flemish Institute for Technological Research (VITO), Boeretang 200, B-2400 Mol, Belgium \\ ${ }^{3}$ Department of Physics, Nanosystems Initiative Munich, and Center for NanoScience, \\ LMU Munich, Amalienstrasse 54, 80799 Munich, Germany \\ ${ }^{4}$ KU Leuven, Division of Molecular Imaging and Photonics, Celestijnenlaan 200F, 3001 Leuven, Belgium
}

(Received 2 September 2016; revised manuscript received 8 December 2016; published 26 May 2017)

\begin{abstract}
Recent magnetic tweezers experiments have reported systematic deviations of the twist response of doublestranded DNA from the predictions of the twistable wormlike chain model. Here we show, by means of analytical results and computer simulations, that these discrepancies can be resolved if a coupling between twist and bend is introduced. We obtain an estimate of $40 \pm 10 \mathrm{~nm}$ for the twist-bend coupling constant. Our simulations are in good agreement with high-resolution, magnetic-tweezers torque data. Although the existence of twist-bend coupling was predicted long ago [J. Marko and E. Siggia, Macromolecules 27, 981 (1994)], its effects on the mechanical properties of DNA have been so far largely unexplored. We expect that this coupling plays an important role in several aspects of DNA statics and dynamics.
\end{abstract}

DOI: 10.1103/PhysRevLett.118.217801

Introduction.-The mechanical properties of doublestranded DNA (dsDNA) are critical for both its structure and function within the cell. The stretching of dsDNA under applied forces has been measured by single molecule techniques $[1,2]$ and is accurately reproduced by a simple polymer model, containing the bending stiffness as the only parameter [3]. Elastic polymer models were also successfully employed to study the torsional properties of dsDNA [4] and compared to single-molecule experiments, such as magnetic tweezers (MT) [5] (Fig. 1, right). The currently accepted elastic model for dsDNA is the twistable wormlike chain (TWLC) [6]. Although the TWLC correctly describes the overall response of dsDNA to applied forces and torques, it fails to quantitatively explain the force dependence of the effective torsional stiffness $[7,8]$. Here, we show that an alternative elastic model proposed by Marko and Siggia (MS) [9] quantitatively describes the force dependence of the effective torsional stiffness, by taking into account a direct coupling between twist and bend deformations. Furthermore, we demonstrate that the MS model explains an unresolved discrepancy in the measured intrinsic torsional stiffness, obtained from different techniques. Finally, we show that the MS model provides a better description of the prebuckling torque response of dsDNA, determined in high-resolution magnetic torque tweezers (MTT) experiments, than the TWLC.

TWLC and MS models.-Both the TWLC and MS models describe dsDNA as a continuous, twistable curve by associating an orthonormal frame $\left\{\hat{\mathbf{e}}_{1}, \hat{\mathbf{e}}_{2}, \hat{\mathbf{e}}_{3}\right\}$ with each base pair (Fig. 1) [9]. We choose $\hat{\mathbf{e}}_{3}$ tangent to the helical axis and $\hat{\mathbf{e}}_{1}$ and $\hat{\mathbf{e}}_{2}$ oriented as in Fig. 1. In the continuum limit these vectors are functions of the arclength variable $s$. For the stretching forces considered here $(f<10 \mathrm{pN})$ dsDNA is inextensible, hence $0 \leq s \leq L$, with $L$ the contour length. A local dsDNA conformation is given by a vector $\boldsymbol{\Omega}(s)$ which describes the infinitesimal rotation connecting $\left\{\hat{\mathbf{e}}_{1}(s), \hat{\mathbf{e}}_{2}(s), \hat{\mathbf{e}}_{3}(s)\right\}$ to $\left\{\hat{\mathbf{e}}_{1}(s+d s), \hat{\mathbf{e}}_{2}(s+d s)\right.$, $\left.\hat{\mathbf{e}}_{3}(s+d s)\right\}$. The direction of $\boldsymbol{\Omega}(s)$ identifies the rotation axis, and $|\boldsymbol{\Omega}(s)| d s$ the infinitesimal rotation angle. In particular, if $\boldsymbol{\Omega}(s)$ is parallel to $\hat{\mathbf{e}}_{3}(s)$, one generates a local rotation along the tangent vector, i.e. a twist deformation. Conversely, an $\boldsymbol{\Omega}(s)$ along $\hat{\mathbf{e}}_{1}(s)$ or $\hat{\mathbf{e}}_{2}(s)$ corresponds to a bending deformation. Expressing the local rotation vector as $\boldsymbol{\Omega}(s)=\sum_{i=1}^{3} \Omega_{i}(s) \hat{\mathbf{e}}_{i}(s)$, one identifies the twist mode with $\Omega_{3}$ and the two bending modes with $\Omega_{1}$ and $\Omega_{2}$.

Marko and Siggia [9] showed that the molecular symmetry of dsDNA imposes only the invariance of the energy to the interchange $\Omega_{1} \rightarrow-\Omega_{1}$. This leads, to lowest order in $\Omega_{i}$, to the following energy functional:

$\beta E_{\mathrm{MS}}=\frac{1}{2} \int_{0}^{L} d s\left(A_{1} \Omega_{1}^{2}+A_{2} \Omega_{2}^{2}+C \Omega_{3}^{2}+2 G \Omega_{2} \Omega_{3}+\cdots\right)$,

where $\beta \equiv 1 / k_{B} T$, and the dots denote higher-order terms. The MS model is characterized by two bending stiffnesses $A_{1}$ and $A_{2}$, a torsional stiffness $C$, and a twist-bend coupling constant $G$, which have dimension of length. Note that twist-bend coupling $\Omega_{2} \Omega_{3}$ is the only quadratic cross term allowed by the $\Omega_{1} \rightarrow-\Omega_{1}$ symmetry [9].

The TWLC is a limiting case of the MS model obtained by setting $A_{1}=A_{2} \equiv A$ and $G=0$

$$
\beta E_{\mathrm{TWLC}}=\frac{1}{2} \int_{0}^{L} d s\left[A\left(\Omega_{1}^{2}+\Omega_{2}^{2}\right)+C \Omega_{3}^{2}+\cdots\right] .
$$


We note that the asymmetric bending stiffness and the twist-bend coupling, which are intrinsic to the MS model and neglected in the TWLC, are naturally suggested by the structure of the DNA helix, with its pronounced minor and major groove (Fig. 1, left). In the following, we analyze the consequences of taking these additional terms into account using analytical calculations and extensive computer simulations. Although the existence of twist-bend coupling was predicted long ago, its effects on the mechanical properties of DNA have been so far largely unexplored. Two studies $[10,11]$ in which the MS model was invoked are discussed below.

Renormalized bending and torsional stiffnesses.-In order to parametrize the MS model, we calculated the renormalized bending and torsional stiffness, $\kappa_{b}$ and $\kappa_{t}$, respectively, from the equilibrium fluctuations of a free chain (see the Supplemental Material [12] for derivation):

$$
\begin{gathered}
\kappa_{\mathrm{b}}=A \frac{1-\frac{\varepsilon^{2}}{A^{2}}-\frac{G^{2}}{A C}\left(1+\frac{\varepsilon}{A}\right)}{1-\frac{G^{2}}{2 A C}}, \\
\kappa_{\mathrm{t}}=C \frac{1-\frac{\varepsilon}{A}-\frac{G^{2}}{A C}}{1-\frac{\varepsilon}{A}},
\end{gathered}
$$

where $A \equiv\left(A_{1}+A_{2}\right) / 2$ is the mean bending stiffness and $\varepsilon \equiv\left(A_{1}-A_{2}\right) / 2$ the bending anisotropy. By setting $\varepsilon=$ $G=0$ one obtains the TWLC values $\kappa_{\mathrm{b}}=A$ and $\kappa_{\mathrm{t}}=C$. Equations (3) and (4) show that in the MS model $\kappa_{\mathrm{b}}<A$ and $\kappa_{\mathrm{t}}<C$; hence, the presence of a direct twist-bend coupling softens the chain, rendering the bending and twisting fluctuations larger than expected from the intrinsic parameters $A$ and $C$, respectively. The details of the parametrization are discussed below. Note, finally, that $\kappa_{\mathrm{b}}$ and $2 \kappa_{\mathrm{t}}$ are also the bending and twisting persistence

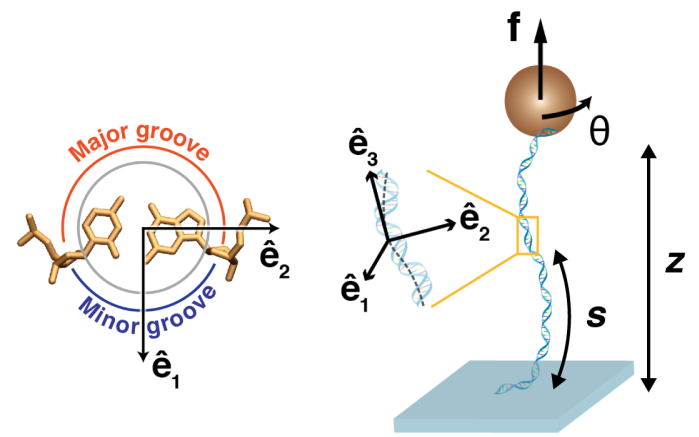

FIG. 1. Schematic representation of a typical MT experiment. Magnetic fields are used to apply forces and torques (inducing a rotation angle $\theta$ ) to a paramagnetic bead. A dsDNA molecule is attached at one side to the bead and at the other to a flow-cell surface, separated by a distance $z$ measuring the extension of the molecule. Continuum elastic models describe the double-helix conformation using an orthonormal frame $\left\{\hat{\mathbf{e}}_{1}, \hat{\mathbf{e}}_{2}, \hat{\mathbf{e}}_{3}\right\}$ at each point along the molecule, labeled by a coordinate $s$. $\hat{\mathbf{e}}_{3}$ is tangent to the helical axis, while $\hat{\mathbf{e}}_{1}$ points from the center of the helix towards the middle of the minor groove, and $\hat{\mathbf{e}}_{2}=\hat{\mathbf{e}}_{3} \times \hat{\mathbf{e}}_{1}$. lengths, that characterize the decay of the respective correlations along the molecule.

Effective torsional stiffness. - The parameter $C$ in Eqs. (1) and (2) is the intrinsic torsional stiffness and quantifies the energetic cost of local pure twist deformations $\left(\Omega_{1}=\right.$ $\Omega_{2}=0, \Omega_{3} \neq 0$ ). The effective torsional stiffness $C_{\text {eff }}$, in contrast, expresses the cost of a global twist deformation, and decreases with decreasing force. The force dependence of $C_{\text {eff }}$ can be understood as follows: In absence of thermal fluctuations a weakly twisted dsDNA is straight, and the twist response is governed by the intrinsic torsional stiffness $C$. In the presence of thermal fluctuations, however, twist can be absorbed by bending [37,38], leading to an effective torsional stiffness $C_{\text {eff }}<C$. High stretching forces suppress bending fluctuations, therefore yielding $z \approx L$ and $C_{\text {eff }} \approx C$, while at low forces fluctuations are large, hence $z<L$ and $C_{\text {eff }}<C$. Moroz and Nelson calculated the force dependence of $C_{\text {eff }}$ for the TWLC in the high-force limit [37]

$$
C_{\text {eff }}=C\left(1-\frac{C}{4 A} \sqrt{\frac{k_{B} T}{A f}}+\cdots\right),
$$

where the dots indicate higher-order corrections in $1 / \sqrt{f}$.

$C_{\text {eff }}$ has been experimentally measured with two independent single-molecule approaches. In magnetic torque tweezers $C_{\text {eff }}$ is obtained from the torque response $\tau$ upon over- and underwinding dsDNA by a small angle $\theta$ (Fig. 1, right) [7,39-41]

$$
\tau \approx \frac{k_{B} T C_{\mathrm{eff}}}{L} \theta
$$

Freely orbiting magnetic tweezers (FOMT) [8] and the rotor bead assay [41,42], in contrast, measure fluctuations of $\theta$ of a freely rotating dsDNA tether, and $C_{\text {eff }}$ is obtained from

$$
C_{\text {eff }}=\frac{L}{\sigma_{\theta}^{2}},
$$

where $\sigma_{\theta}^{2}$ is the variance of $\theta$. MTT and FOMT yield consistent values of $C_{\text {eff }}$, which, however, deviate from the TWLC prediction of Eq. (5) [7,8] (Fig. 2).

To investigate the force dependence of $C_{\text {eff }}$, we performed computer simulations of the TWLC and the MS models using a coarse-grained model, similar to Ref. [43], where the dsDNA is represented by a series of connected beads, each carrying an orthonormal frame of reference. Successive beads interact via potential energies obtained from the discretization of Eqs. (1) or (2) in the MS or TWLC models, respectively [12]. The effect of an implicit solvent was introduced via Langevin forces [44].

The TWLC simulations with $A=43 \mathrm{~nm}$ and $C=110 \mathrm{~nm}$ (Fig. 2, open circles) are in excellent agreement with the high-force expansion of Eq. (5), (Fig. 2, solid line). This is 


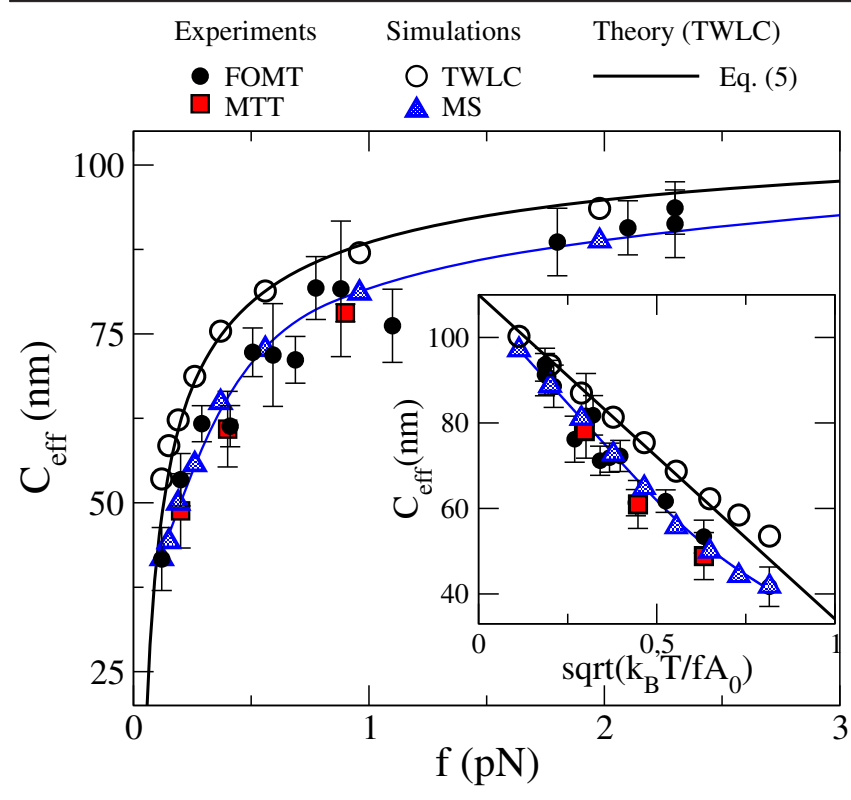

FIG. 2. Force dependence of the effective torsional stiffness from MTT (present work) and FOMT [8] measurements, from simulations of the TWLC and the MS models (where error bars are smaller than the symbols) and from the analytical TWLC approximation [Eq. (5)]. Parameters are $A=43 \mathrm{~nm}$ and $C=$ $110 \mathrm{~nm}$ for the TWLC and $A=56 \mathrm{~nm}, C=110 \mathrm{~nm}$, $\varepsilon=10 \mathrm{~nm}$, and $G=40 \mathrm{~nm}$ for the MS model. The inset shows $C_{\text {eff }}$ as a function of the rescaled variable $\sqrt{k_{B} T / A_{0} f}$ (with $A_{0}=50 \mathrm{~nm}$ ); in these units Eq. (5) becomes a straight line. The experimental data are not well described by the TWLC, but agree quantitatively with the MS model (reduced $\chi_{\text {TWLC }}^{2}=6.1$ and $\chi_{\text {MS }}^{2}=0.74$, respectively). The blue line is an interpolation of the MS simulations points.

a good test of our simulations and shows that higher-order corrections to (5) do not contribute significantly to $C_{\text {eff }}$. The value of $A=43 \mathrm{~nm}$ comes from the measured persistence length in Ref. [8] while the value $C=110 \mathrm{~nm}$ of the intrinsic torsional stiffness has been obtained from extrapolation of $C_{\text {eff }}$ to high forces (see inset of Fig. 2 and [12]).

We then turned to the MS model, which was parametrized as follows: Similarly to the TWLC, the intrinsic torsional stiffness was set to $C=110 \mathrm{~nm}$. Following Ref. [45], we chose $\varepsilon=10 \mathrm{~nm}$ for the bending anisotropy (tests for different values of $\varepsilon$ are shown in the Supplemental Material [12]). $A$ and $G$ were chosen so that Eq. (3) gives $\kappa_{\mathrm{b}}=43 \mathrm{~nm}$, the measured persistence length of dsDNA. Therefore only one of the two could be freely adjusted. The best fit to the data was found for $G=40 \mathrm{~nm}$ and $A=56 \mathrm{~nm}$, and is in quantitative agreement with the experiments. Control simulations for other values of $\varepsilon$ and $\kappa_{\mathrm{b}}$ gave similar estimates of the twist-bend stiffness [12]. We finally obtained $G=40 \pm 10 \mathrm{~nm}$, where the error indicates the range of values for which simulations fit the MT data within their experimental errors.

This value is somewhat higher than the estimate $G=25 \mathrm{~nm}$ [11], obtained from the analysis of structural correlations of dsDNA wrapped around a histone core. Elastic couplings in dsDNA were also investigated in allatom simulations $[10,46]$. These and more recent studies [47] show that the twist-bend coupling is the most significant among the off-diagonal elastic terms (i.e. $\Omega_{1} \Omega_{2}, \Omega_{1} \Omega_{3}$, and $\Omega_{2} \Omega_{3}$ ), in agreement with the symmetry analysis by Marko and Siggia [9].

Intrinsic torsional stiffness. - The experimental determination of the intrinsic torsional stiffness $C$ has proven to be a challenging task, with different experimental techniques yielding values within the range 40-120 nm [7,38,40, 48-53]. The techniques used for this purpose can be divided into two main categories. The first group contains single-molecule techniques as MT, in which a stretching force is applied to DNA. One can obtain $C$ from high-force extrapolation of $C_{\text {eff }}$, which typically yields values in the range of 100-110 nm [7,51-53]. In the second group of techniques no force is applied to the DNA molecule, as in fluorescence polarization anisotropy $[48,49]$, the analysis of cyclization rates [54], or topoisomer distributions [55]. Typical values from these studies lie in the range of 60-80 nm [12].

According to the TWLC all the above techniques should probe the intrinsic torsional stiffness $C$. In the framework of the MS model, however, this is not the case; the torsional response at high tension is still governed by $C$, since in this limit bending fluctuations are suppressed, i.e. $\Omega_{1}, \Omega_{2} \rightarrow 0$. In absence of applied forces, however, bending fluctuations influence the measured torsional stiffness via the twistbend coupling $G$, leading to twist stiffness $\kappa_{\mathrm{t}}<C$ according to Eq. (4). With the parametrization used for the fit of Fig. $2(A=56 \mathrm{~nm}, \varepsilon=10 \mathrm{~nm}, C=110 \mathrm{~nm}$, and $G=40 \mathrm{~nm})$, Eq. (4) gives $\kappa_{\mathrm{t}}=75 \mathrm{~nm}$, which is consistent with the values obtained from the second family of techniques (details in Supplemental Material [12]). We conclude that the wide spread in the experimental $C$ values, which appears to be a discrepancy in the TWLC model, is naturally explained within the framework of the MS model.

Extension and torque.-To further investigate the effect of twist-bend coupling, we performed high-resolution MTT extension and torque measurements and compared to simulations of the TWLC and MS models. We introduce the supercoiling density $\sigma \equiv \theta /\left(\omega_{0} L\right)$, where $\omega_{0} \approx$ $1.85 \mathrm{~nm}^{-1}$ is the intrinsic twist density of dsDNA. Although the discussion so far has been limited to the regime of small $|\sigma|$, we now extend our analysis to high values of $|\sigma|$, for which dsDNA undergoes a buckling transition to interwound structures called plectonemes. We will first discuss the prebuckling (low $|\sigma|$ ) and then the postbuckling (high $|\sigma|$ ) regime.

Figures 3(a) and 3(b) show the $\sigma$ dependence of the relative extension $z / L$ and torque $\tau$, respectively, for $f=0.4 \mathrm{pN}$ (similar plots for $f=0.2$ and $0.9 \mathrm{pN}$ are in the Supplemental Material [12]). The shaded area in Fig. 3 indicates the prebuckling regime, in which the simulated 


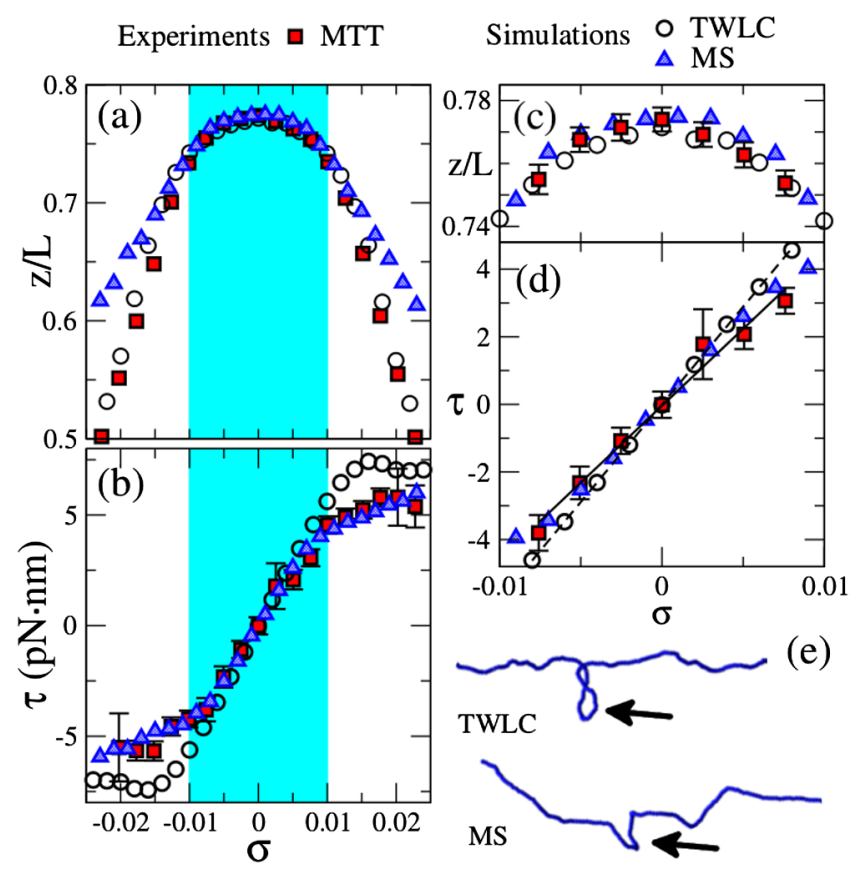

FIG. 3. (a) Relative extension $z / L$ and (b) torque $\tau$ vs supercoiling density $\sigma$ at $f=0.4 \mathrm{pN}$ from MTT experiments (solid squares) and simulations of the TWLC (open circles, $A=43 \mathrm{~nm}, C=110 \mathrm{~nm}$ ) and MS (solid triangles, $A=56 \mathrm{~nm}$, $C=110 \mathrm{~nm}, \quad G=40 \mathrm{~nm}, \quad$ and $\varepsilon=10 \mathrm{~nm}$ ) models. (c), (d) Closeups of $z / L$ and $\tau$ in the prebuckling regime, shaded area in (a) and (b). (e) Snapshots of simulations of the TWLC and MS models, respectively, at $\sigma=0.023$. The arrows point to a plectonemic supercoil in the TWLC and to a solenoidal supercoil in the MS model.

values of $z / L$ for the two models differ by less than $1 \%$ [Fig. 3(c)]. In addition, both models are consistent with the MTT measurements of the dsDNA extension (reduced $\chi_{\mathrm{TWLC}}^{2}=1.6$ and $\chi_{\mathrm{MS}}^{2}=0.77$, averaged over all forces). In contrast, there is a noticeable difference between the models in the behavior of the torque at small $|\sigma|$ [Fig. 3(d)], with the MS model providing a significantly better prediction of the experimental data than the TWLC (reduced $\chi_{\mathrm{TWLC}}^{2}=5.9$ and $\chi_{\mathrm{MS}}^{2}=1.3$, again averaged over all forces). The quantitative agreement of the MS model with experimental extension and torque data in the prebuckling regime further highlights the importance of including the twist-bend coupling term in elastic models of dsDNA, and provides an independent test of our estimate of $G$.

We now focus on the postbuckling regime, where simulations of the TWLC show that plectonemes form at sufficiently high $|\sigma|$ [Fig. 3(e)] and that the relative extension data fit well the experiments [Fig. 3(a)], in agreement with previous work [56-58]. However the TWLC fails to account for the torque data, both in the prebuckling, as already discussed, and even more clearly in the postbuckling regime [Fig. 3(b)]. Our work highlights the need for direct torque measurements to quantitatively test elastic models of DNA.
Earlier comparisons between analytical predictions and experiments were limited to extension data $[38,53]$, since only in the past few years direct torque measurements have become available [7,40-42,52].

The postbuckling behavior of the MS is somehow complementary to that of the TWLC. The torque is in quantitative agreement with the experiments, but there are deviations in the extension. Figure 3(e) shows simulation snapshots of equilibrium conformations for the TWLC and MS models at $\sigma=0.023$. Whereas the TWLC forms a plectoneme, the MS model favors highly twisted helical configurations, known as solenoidal supercoils [9]. This different behavior can be explained as follows: Upon twisting, there is an energetic penalty in the TWLC, due to $C \Omega_{3}^{2}$ in Eq. (2). Beyond a threshold value of $|\sigma|$, part of the twist is transformed into a localized bending deformation, giving rise to plectonemic supercoils [Fig. 3(e)]. On the other hand, in the MS model (1), the torsional energy can be reduced by a uniform bending such that the quantity $\left\langle\Omega_{2} \Omega_{3}\right\rangle$ becomes negative. This gives rise to the characteristic helical structures [solenoidal supercoils, Fig. 3(e)]. The absence of plectonemes in the range of $\sigma$ considered is a shortcoming of the MS model, as defined by the energy functional (1), and is the reason why $z / L$ decreases with $|\sigma|$ less steeply than in the experiments.

Thus, we conclude that at high $\sigma$ both models deviate from experiments, although in different ways. It should be stressed that both models are obtained as quadratic expansions in the deformation parameters $\Omega_{i}$. It is likely that, close to buckling, higher-order anharmonic terms in $\Omega_{i}$ will become relevant. This is particularly true for the MS model, where the molecular asymmetry of dsDNA allows six additional third-order terms: $\Omega_{1}^{2} \Omega_{2}, \Omega_{1}^{2} \Omega_{3}, \Omega_{2}^{2} \Omega_{3}, \Omega_{2} \Omega_{3}^{2}$, $\Omega_{2}^{3}$, and $\Omega_{3}^{3}$ [9].

Discussion.-We investigated the mechanical properties of an elastic DNA model with an explicit twist-bend coupling [9]. Our analysis focused on the regime where the supercoiling density is small; i.e., twist and bending deformations are weak. We showed that the model resolves two issues that the standard TWLC fails to explain (i) the force dependence of the effective torsional stiffness, also discussed in the recent literature $[7,8,57,59]$ and (ii) discrepancies in the reported estimates of the intrinsic torsional stiffness $[48,49,53]$. Moreover, it provides a superior fit to high-resolution torque data, compared to the TWLC.

An alternative model, that invokes a cooperative structural transition in the DNA helix, was recently proposed by Schurr [59]. While this model also explains the deviations between the $C_{\text {eff }}$ data and the TWLC predictions, we note that our current model naturally follows from the molecular symmetry of the DNA helix [9] and independently explains several different features of the torsional response of DNA.

Although we showed that the MS model is a more accurate mechanical model of dsDNA than the standard TWLC in the prebuckling regime, there is still an open 
issue at high $|\sigma|$, where neither of the two models fully agrees with the experiments. The MS model, as defined by the energy functional of Eq. (1), incorrectly predicts a strong relative preference for solenoidal supercoils over plectonemes. We believe this could possibly be resolved by introducing higher-order terms. In future work, it would be interesting to extend the MS model to account for all experimental observables even in the high $|\sigma|$ regime. Twistbend coupling could influence, for instance, the long-range transfer of supercoiling density [60] and the interactions with proteins which bend and twist DNA [61,62].

We thank M. Schurr and F. Lankaš for useful discussions and the KU Leuven grant IDO/12/08, the Research Funds Flanders (FWO Vlaanderen), the Deutsche Forschungsgemeinschaft through Grant No. SFB863 for financial support.

[1] S. B. Smith, L. Finzi, and C. Bustamante, Science 258, 1122 (1992).

[2] C. Bustamante, Z. Bryant, and S. B. Smith, Nature (London) 421, 423 (2003).

[3] C. Bustamante, J. Marko, E. Siggia, and B. Smith, Science 265, 1599 (1994).

[4] J. F. Marko and E. D. Siggia, Science 265, 506 (1994).

[5] T. Strick, J.-F. Allemand, D. Bensimon, A. Bensimon, and V. Croquette, Science 271, 1835 (1996).

[6] P. Nelson, M. Radosavljevic, and S. Bromberg, Biological Physics: Energy, Information, Life (W.H. Freeman and Co., New York, 2002).

[7] J. Lipfert, J. W. Kerssemakers, T. Jager, and N. H. Dekker, Nat. Methods 7, 977 (2010).

[8] J. Lipfert, M. Wiggin, J. W. Kerssemakers, F. Pedaci, and N. H. Dekker, Nat. Commun. 2, 439 (2011).

[9] J. Marko and E. Siggia, Macromolecules 27, 981 (1994).

[10] F. Lankaš, J. Šponer, P. Hobza, and J. Langowski, J. Mol. Biol. 299, 695 (2000).

[11] F. Mohammad-Rafiee and R. Golestanian, Phys. Rev. Lett. 94, 238102 (2005).

[12] See Supplemental Material at http://link.aps.org/ supplemental/10.1103/PhysRevLett.118.217801, which contains Refs. [13-36], for a detailed derivation of $\kappa_{\mathrm{b}}$ and $\kappa_{\mathrm{t}}$, additional tests of the MS model, and details about experiments and simulations.

[13] B. Eslami-Mossallam and M. Ejtehadi, J. Chem. Phys. 128, 125106 (2008).

[14] V. V. Rybenkov, N. R. Cozzarelli, and A. V. Vologodskii, Proc. Natl. Acad. Sci. U.S.A. 90, 5307 (1993).

[15] S. Plimpton, J. Comp. Physiol. 117, 1 (1995).

[16] J. F. Marko and E. D. Siggia, Macromolecules 28, 8759 (1995).

[17] J. R. Wenner, M. C. Williams, I. Rouzina, and V. A. Bloomfield, Biophys. J. 82, 3160 (2002).

[18] J. Lipfert, X. Hao, and N. H. Dekker, Biophys. J. 96, 5040 (2009).

[19] E. Herrero-Galan, M. E. Fuentes-Perez, C. Carrasco, J. M. Valpuesta, J. L. Carrascosa, F. Moreno-Herrero, and J. R. Arias-Gonzalez, J. Am. Chem. Soc. 135, 122 (2013).
[20] J. F. Marko, Physica (Amsterdam) 418A, 126 (2015).

[21] T. Odijk, J. Polym. Sci., Polym. Phys. Ed. 15, 477 (1977).

[22] J. Skolnick and M. Fixman, Macromolecules 10, 944 (1977).

[23] J. L. Barrat and J. F. Joanny, Europhys. Lett. 24, 333 (1993).

[24] A. J. Te Velthuis, J. W. Kerssemakers, J. Lipfert, and N. H. Dekker, Biophys. J. 99, 1292 (2010).

[25] C. Gosse and V. Croquette, Biophys. J. 82, 3314 (2002).

[26] I. Vilfan, J. Lipfert, D. Koster, S. Lemay, and N. Dekker, in Handbook of Single-Molecule Biophysics (Springer, New York, 2009), pp. 371-395.

[27] L. Oroszi, P. Galajda, H. Kirei, S. Bottka, and P. Ormos, Phys. Rev. Lett. 97, 058301 (2006).

[28] J. C. Gore, Ph.D. thesis, University of California, 2005.

[29] T. Strick, D. Bensimon, and V. Croquette, Genetica (The Hague) 106, 57 (1999).

[30] A. V. Vologodskii and J. F. Marko, Biophys. J. 73, 123 (1997).

[31] D. Shore and R. L. Baldwin, J. Mol. Biol. 170, 957 (1983).

[32] W. H. Taylor and P. J. Hagerman, J. Mol. Biol. 212, 363 (1990).

[33] A. V. Vologodskii, V. V. Anshelevich, A. V. Lukashin, and M. D. Frank-Kamenetskii, Nature (London) 280, 294 (1979).

[34] P. R. Selvin, D. N. Cook, N. G. Pon, W. R. Bauer, M. P. Klein, and J. E. Hearst, Science 255, 82 (1992).

[35] P. J. Heath, J. B. Clendenning, B. S. Fujimoto, and M. J. Schurr, J. Mol. Biol. 260, 718 (1996).

[36] I. Hurley, P. Osei-Gyimah, S. Archer, C. P. Scholes, and L. S. Lerman, Biochemistry 21, 4999 (1982).

[37] J. D. Moroz and P. Nelson, Proc. Natl. Acad. Sci. U.S.A. 94, 14418 (1997).

[38] C. Bouchiat and M. Mézard, Phys. Rev. Lett. 80, 1556 (1998).

[39] J. Lipfert, G. M. Skinner, J. M. Keegstra, T. Hensgens, T. Jager, D. Dulin, M. Köber, Z. Yu, S. P. Donkers, F.-C. Chou, R. Das, and N. H. Dekker, Proc. Natl. Acad. Sci. U.S.A. 111, 15408 (2014).

[40] D. J. Kauert, T. Kurth, T. Liedl, and R. Seidel, Nano Lett. 11, 5558 (2011).

[41] F. C. Oberstrass, L. E. Fernandes, and Z. Bryant, Proc. Natl. Acad. Sci. U.S.A. 109, 6106 (2012).

[42] Z. Bryant, M. D. Stone, J. Gore, S. B. Smith, N. R. Cozzarelli, and C. Bustamante, Nature (London) 424, 338 (2003).

[43] C. Brackley, A. Morozov, and D. Marenduzzo, J. Chem. Phys. 140, 135103 (2014).

[44] R. Zwanzig, Nonequilibrium Statistical Mechanics (Oxford University Press, Oxford, England, 2001).

[45] D. Norouzi, F. Mohammad-Rafiee, and R. Golestanian, Phys. Rev. Lett. 101, 168103 (2008).

[46] F. Lankaš, J. Šponer, J. Langowski, and T. E. Cheatham, Biophys. J. 85, 2872 (2003).

[47] T. Dršata, N. Špačková, P. Jurečka, M. Zgarbová, J. Šponer, and F. Lankaš, Nucleic Acids Res. 42, 7383 (2014).

[48] J. H. Shibata, B. S. Fujimoto, and J. M. Schurr, Biopolymers 24, 1909 (1985).

[49] B. S. Fujimoto and J. M. Schurr, Nature (London) 344, 175 (1990).

[50] Z. Bryant, F. C. Oberstrass, and A. Basu, Curr. Opin. Struct. Biol. 22, 304 (2012).

[51] F. Mosconi, J. F. Allemand, D. Bensimon, and V. Croquette, Phys. Rev. Lett. 102, 078301 (2009). 
[52] S. Forth, C. Deufel, M. Y. Sheinin, B. Daniels, J. P. Sethna, and M. D. Wang, Phys. Rev. Lett. 100, 148301 (2008).

[53] J. D. Moroz and P. Nelson, Macromolecules 31, 6333 (1998).

[54] S. D. Levene and D. M. Crothers, J. Mol. Biol. 189, 61 (1986).

[55] D. Shore and R. L. Baldwin, J. Mol. Biol. 170, 983 (1983).

[56] R. Schöpflin, H. Brutzer, O. Müller, R. Seidel, and G. Wedemann, Biophys. J. 103, 323 (2012).
[57] T. Lepage, F. Képès, and I. Junier, Biophys. J. 109, 135 (2015).

[58] I. D. Ivenso and T. D. Lillian, Biophys. J. 110, 2176 (2016).

[59] J. M. Schurr, J. Phys. Chem. B 119, 6389 (2015).

[60] M. van Loenhout, M. de Grunt, and C. Dekker, Science 338, 94 (2012).

[61] Y. Kim, J. H. Geiger, S. Hahn, and P. B. Sigler, Nature (London) 365, 512 (1993).

[62] W. Vanderlinden, J. Lipfert, J. Demeulemeester, Z. Debyser, and S. De Feyter, Nanoscale 6, 4611 (2014). 